\title{
Retinoblastoma cM1a TNM Finding v7
}

National Cancer Institute

\section{Source}

National Cancer Institute. Retinoblastoma cM1a TNM Finding v7. NCI Thesaurus. Code C88778.

Single lesion to sites other than CNS. (from AJCC 7th Ed.) 